\title{
Redes sociales, tejidos de paz
}

\author{
Oscar Yecid Aparicio Gómez* \\ Olga Lucía Ostos Ortiz* \\ Mauricio Cortés Gallego ${ }^{* * *}$
}

\begin{abstract}
Recibido: 12 de octubre del 2018
Evaluado: 8 de noviembre de 2018

Aceptado: 14 de noviembre del 2018
\end{abstract}

Citar como: Aparicio Gómez, O. Y., Ostos Ortiz, O. L. y Cortés Gallego, M. (2019). Redes sociales, tejidos de paz. Hallazgos, 16(32), 17-25. DOI: https:/l doi.org/10.15332/2422409X.4999

\section{RESUMEN}

Los nuevos lenguajes, cada vez con mayor procedencia de la inmersión tecnológica a que se ha sometido voluntariamente la sociedad actual, requieren ser analizados desde las perspectivas que generan las nuevas maneras de relacionarse. No es extraño encontrarse conectado simultáneamente a diversas redes en un espacio común; los criterios que se tienen en cuenta para incorporar o invitar interlocutores a estos espacios comunes, y que deberían responder a los gustos e intereses de todos, no siempre resultan según el deber ser. El trabajo de construcción de relaciones interpersonales a través de herramientas comunicativas en línea hace posible tejer redes, no solo para construir relaciones, sino para que a través de ellas se pueda genera nuevo conocimiento. Las experiencias en red que permiten identificar los esfuerzos para gestar una sociedad en paz requieren de atención y cuidados específicos para que la construcción que se consiga prescinda de los vicios que atentan contra la durabilidad que todas las personas desean en sus relaciones interpersonales.

Palabras clave: comunicación, persona, redes sociales, conectivismo.

Doctor en Filosofía (2006) y doctor en Educación (2015) de la Universidad de Barcelona, España. Profesor y editor. Bogotá D. C., Colombia. Correo electrónico: oscar.yecid@gmail.com - ORCID: 0000-0003-3535-6288.

* Magíster en Genética, Universidad Nacional de Colombia. Directora Unidad de Investigación, Universidad Santo Tomás, Bogotá D. C., Colombia. Correo electrónico: olgaostosortiz@gmail.com - ORCID: https://orcid.org/0000-0002-6477-9872.

... Vicerrector académico de la Universidad Santo Tomás, Bogotá D. C., Colombia. Correo electrónico: viceacadgenera@@usantotomas.edu.co - ORCID: https://orcid.org/0000-0002-9446-8389. 


\title{
Social networks, fabrics of peace
}

\begin{abstract}
New languages, from immersion technology to which today's society has voluntarily submitted, need to be analyzed from the perspectives generated by new ways of relating. So it is not unusual to find several networks simultaneously connected in a common space; the criteria taken into account to include or invite partners to these common spaces, and that should respond to the tastes and interests of all, do not always result as it ought to be. The work of building relationships through online communication tools makes it possible to create networks, not only to build relationships, but to generate new knowledge through them. Networking experiences that identify efforts to create a peaceful society require specific attention and care so the construction achieved leaves out the vices that threaten the durability that everyone desires in their interpersonal relationships.
\end{abstract} Received: october 12, 2018 Evaluated: november 8, 2018. Accepted: november 14, 2018

Keywords: Communication, person, social networks, connectivism. 


\section{Redes sociais, tecidos de paz}

Recebido: 12 de outubro de 2018 Avaliado: 8 de novembro de 2018 Aceito: 14 de novembro de 2018

\section{Resumo}

As novas linguagens, cada vez com maior procedência da imersão tecnológica que tem se submetido voluntariamente a sociedade atual requerem ser analisadas desde as perspectivas que geram as novas formas de se relacionar. Não é estranho estar conectado simultaneamente às diversas redes em um espaço comum; os critérios que se levam em consideração para incorporar ou convidar interlocutores nesses espaços comuns e que deveriam respondes aos gostos e interesses de todos, não sempre resultam segundo o dever ser. O trabalho de construção de relações interpessoais através de ferramentas comunicativas online faz possível tecer redes, não só para construir relações, senão para que através delas poda-se gerar novo conhecimento. As experiências na rede que permitem identificar os esforços para gestar uma sociedade em paz requerem de atenção e cuidados específicos para que a construção alcançada prescinda dos vícios que atentam contra a durabilidade que todas as pessoas desejam nas suas relações interpessoais.

Palavras-chave: comunicação, pessoa, redes sociais, conectivismo . 


\section{INTRODUCCIÓN}

En los últimos años la tecnología ha reconfigurado la manera en que se relacionan las personas, y en un período muy breve de tiempo ha impregnado la mayoría de las interacciones humanas con su entorno. Las nuevas formas de aprender no han sido ajenas a esta presencia, casi omnímoda, de las condiciones que facilitan el acceso a la información, no siempre mediadas por un adecuado acompañamiento en la filtración y selección de contenidos acordes a las necesidades de búsqueda de las personas según sus intereses (Aparicio, 2018).

Los lenguajes emergentes de este tipo de relaciones se han incorporado en la sociedad actual de manera exponencial. No es ajeno para la mayoría de las personas que se ha pasado, casi sin esperar apenas un lapso de tiempo, de una comunicación directa cara a cara a una habitual comunicación próxima o remota verbal, y últimamente, próxima o remota escrita, sin que el cara a cara resulte ya relevante.

Estas nuevas dinámicas comunicativas han permeado con fuerza y rapidez en las generaciones más jóvenes, sin que sus predecesores tengan apenas tiempo de considerar y estimar la pertinencia de los nuevos hallazgos en materia de comunicación en este sentido. Las personas que han nacido en las dos últimas décadas, y que acceden a este tipo de tecnologías, desarrollan unas prácticas comunicativas que procesan altos niveles de información con diversos interlocutores de manera simultánea y en períodos muy breves de tiempo, sin que el espacio represente ninguna limitación.

\section{Múltiples Conexiones SIMULTÁNEAS, UN ÚNICO ESPACIO}

La percepción de tiempo y espacio como condiciones de posibilidad para la existencia física, y en el caso de los fenómenos psíquicos, la dimensión temporal (Kant, 2005), comienzan a reconfigurarse a través de las relaciones humanas mediadas por la tecnología digital. Esta multiplicidad sincrónica espacial que las formas de comunicación actual generan en las relaciones humanas se asimilan multimodalmente en el diario vivir de las sociedades alrededor del mundo.

De esta manera, emergen nuevas formas de comunicación que aparecen tan rápido como evolucionan en entramados cada vez más complejos. Se ha pasado del correo postal al correo electrónico, de la traducción reglada a los traductores de bolsillo, de la gramática correcta al uso de símbolos y abreviaciones, de los procesos lentos a procesamientos multitarea medidos en micro y nanosegundos; y todos tienen en común su redefinición de las categorías de tiempo y espacio.

Se vive muy rápido, se dice todo muy rápido y a muchas personas a la vez. Se escribe hoy y no se recuerda mañana. Se lee hoy y mañana se olvida. Se vive al día. Se lee al día. Se escribe al día. Las nuevas formas de lectura y escritura exigen pensar lento y hablar o escribir rápido. Se dice aquí y se lee allá, se escribe aquí y se comenta allá, a ultramar.

Las relaciones humanas crecen, al menos en número, en intensidad de mensajes, de reacciones, de comentarios. Se otorga poder a las redes, poder para ser reconocido o ignorado, la calidad y alcance de las ideas y comentarios por escrito o a través de mensajes 
de voz o videos pueden generar cambios importantes en cada persona y en su entorno.

\section{Elegir PARA CONECTAR}

Los criterios para invitar a otra persona a pertenecer a las propias redes tienen mucho que ver con los gustos o las aficiones, pero cada vez tienen más que ver con la confianza. El número creciente de situaciones adversas, desagradables e incómodas que se viven en ambientes digitales son fáciles de solucionar con solo oprimir un botón. Sin embargo, no todas las personas tienen la capacidad para tomar una decisión correcta y contundente.

El desarrollo de la consciencia moral (Kohlberg, 1992) de la niñez y juventud que tenemos el deber de proteger y promover, se sacude entre el reconocimiento de la propia identidad y la necesidad de encajar en medio de su grupo social más próximo. La lucha interior que se genera al tener que vivir en medio de estas dos realidades, la frustración de ceder en ocasiones para mantener un clima favorable en las relaciones humanas, lleva algunas veces a tomar decisiones equivocadas.

El acompañamiento de entornos cercanos, tales como la familia y la escuela, es imprescindible a la hora de asegurar la toma de decisiones más ajustadas a las necesidades específicas de niños y jóvenes. La seguridad que genera una comunicación basada en la confianza y en la presencia incondicional de los adultos puede hacer más asertivas las elecciones que se ordenan a una opción fundamental desde la experiencia de lo correcto, lo bueno, lo justo (Aparicio y Ostos, 2018a).

Estos criterios generales actúan como soporte de los criterios particulares de las personas necesitadas de un acompañamiento especial, por razón de su edad e inexperiencia. La pedagogía debe impregnar las elecciones vitales de una persona; la paciencia y el acompañamiento en los procesos de conocimiento de otras personas y su incorporación al quehacer habitual de cada uno luego de haber cumplido una serie de etapas, permite asegurar la construcción de una vida menos proclive a caer en el engaño, la idealización o la desesperación.

\section{TEJer LA RED}

El concepto de red supone su comprensión en términos de tejido. No se puede hablar de redes si no se han tejido previamente. El encuentro con otra persona sugiere construcción, de conversaciones, de experiencias, de relatos compartidos; se construye, se teje conocimiento, compartido, relatado, vivido. El ser humano, o teje con otros las redes compartidas de las relaciones interpersonales, o no hay dónde soportar el propio peso de la existencia.

Estas relaciones construyen vidas, y también construyen conocimiento. Una sola persona no lo puede experimentar todo, gracias a las otras personas se puede completar aquello que no se tiene a disposición: la experiencia de la comunidad. El conocimiento está en el otro, en lo otro, y en la experiencia de lo otro en el otro. El diálogo hace posible el intercambio de la experiencia personal de ese conocimiento que reside en aquel que es como yo pero siendo él.

El propio conocimiento se alberga en el otro y viceversa. La sinergia de ambos enriquece a quienes se vinculan a esas experiencias compartidas. Y se inicia el tejido. La razón 
de ser de los nodos son las conexiones, y de las neuronas las sinapsis; sin la conexión se perdería la funcionalidad de cada unidad. El ser humano está diseñado para establecer conexiones a través de los sentidos, conexiones de ida y vuelta, que llevan y traen, que permiten entrar y salir de mundos previamente conectados a otros mundos, de enriquecerse entre todos.

A medida que los nodos se enriquecen pueden ofrecer más a los otros, y se harán más conexiones, todos querrán conectarse a ellos. La percepción general sobre las conexiones tiene que ver con las conexiones endogámicas, reiterativas, quísticas y cerradas en realidades muy específicas. Las conexiones exogámicas enriquecen las conexiones, generan tejidos más sólidos y permiten la construcción de redes consistentes y con mayor impacto.

En este sentido, Siemens (2005) propone desde el conectivismo una alternativa para fortalecer las redes interpersonales en orden al aprendizaje permanente, teniendo en cuenta que el enriquecimiento del conocimiento depende de la diversidad de fuentes, de buscar esas fuentes y luego de aprender la lección ofrecida por ellas, para ponerla a disposición de otras personas. Por otra parte, se destaca el hecho de nutrir las redes para nutrir a las personas que acceden a ellas, y de igual manera ofrecer a las personas el acceso a las redes para nutrir las demás redes; esta actualización permanente permitirá crear conexiones entre todas las áreas, personas, países, culturas y acceder al conocimiento transdisciplinar que privilegie la conservación de la vida en todas sus manifestaciones.

\section{EXPERIENCIAS EN RED}

En este contexto, ponerse delante de una pantalla exige una actitud propositiva, que estimule al crecimiento de quienes se encuentran del otro lado, de muchos lados, con pensamientos distintos. Se requiere aportar a los demás desde la convicción, pero con un discurso inclusivo; desde la diversidad, pero valorando al interlocutor por quien es, no por lo que representa; desde el reconocimiento, pero en las mismas condiciones para todas las personas.

Las redes sociales en entornos virtuales permiten abrir la comprensión humana al reconocimiento del otro como persona, con necesidades similares a los demás interlocutores, con proyectos tan grandes como se pueda sentir soportado por sí mismo y por las demás personas. El acceso casi irrestricto a diversos ambientes puede generar un impacto determinante en la construcción social (Aparicio y Ostos, 2018b).

La paz es un tejido, tejido social, se teje entre todos. La red social es el resultado de tejido social. El lenguaje, la manera de decir las cosas, los criterios para decidir qué decir y qué callar, qué decir de una manera o callar de otra, son cruciales a la hora de construir, de tejer escenarios de paz. Los malentendidos proceden, en su mayoría, de problemas de lenguaje; decir las cosas bien, entenderlas bien, decodificar correctamente el lenguaje del interlocutor es determinante para estar bien, para sentirse bien, para vivir bien.

La responsabilidad de quien escribe es casi más importante que la de aquel quien lee. El uso de las redes sociales como espacios de conciliación, de concertación, de no descalificación, representa un reto inaplazable. La 
interacción diaria que comporta el uso de la tecnología no exime de pensar bien lo que se escribe, lo que se responde, lo que se apoya y lo que simplemente se pasa desapercibido.

\section{Aprendizajes en CONTEXTOS EMERGENTES}

Los referentes pedagógicos ligados a procesos educativos de acompañamiento del aprendiz se hacen reconocibles a partir de la actividad, considerada como el origen del desarrollo cognitivo (Piaget, 1980). La actividad proporciona una seguridad especial en la construcción de los ambientes donde se fragua el aprendizaje; ir de un lugar a otro, últimamente en términos de no lugar, representa un desafío al tránsito cognitivo de la ignorancia al saber. Las nuevas generaciones asumen la no extensión física, verbal o escrita como un presupuesto habitual a la hora de aprender y de comunicarse; no obstante, para otro tipo de aprendizajes más enraizados a la corporalidad, como la presencia física y la interacción verbal, las nuevas interacciones representan un desafío.

La disposición al uso de nuevas herramientas cognitivas acelera el aprendizaje de nuevas maneras de comunicarse a lo largo de la vida, mediante el acceso a fenómenos complejos a través de procesos simples mediados por lenguajes innovadores (Papert, 1982). Esta nueva configuración lingüística requiere el supuesto de la interacción entre un aprendizaje personal y social, regulado especialmente por la colaboración desde una doble dimensión: colaborar para aprender y aprender para colaborar (Dewey, 1938).

En este contexto, la escuela comienza a ser reconocida cada vez más como una tecnología de la educación, contribuyendo a aquello que la cultura y la sociedad reconocen como inteligencia (Lalueza, Crespo y Camps, 2008). Si bien el aprendizaje de este tipo, denominado formal, no se agota en sí mismo, sino que forma parte de un ecosistema que incluye la no formalidad entendida como no escolar o universitario, y la informalidad que permea el proceso educativo, principalmente por la exposición permanente a los medios de comunicación social.

El acceso a otras tecnologías que facilitan el aprendizaje de las personas, en contextos informales, incluyen también las TIC, y específicamente las redes sociales como reflejo del mundo interconectado donde "los nodos compiten siempre por conexiones, porque los enlaces representan supervivencia en un mundo interconectado. (...) Los nodos que adquieren un mayor perfil serán más exitosos en adquirir conexiones adicionales" (Barabási, 2002, p. 107).

\section{LAS REDES SOCIALES COMO HERRAMIENTAS COGNITIVAS}

El aprendizaje en contextos no regulados o informales, especialmente aquellos que se fraguan en ambientes no presenciales, requiere considerar las herramientas tecnológicas-incluyendo las redes sociales-como herramientas cognitivas que hacen posible aprender con la tecnología (Jonassen, 2000), moldear el pensamiento como herramientas amplificadoras (Rheingold, 1985) y reorganizadoras de la mente (Pea, 1985), y construir nuevos aprendizajes actuando como andamios cognitivos (Bruner, 1981).

Las redes sociales tienen un gran potencial en la educación, especialmente porque 
impulsan y vinculan de una manera particular a los estudiantes con su propio aprendizaje (Valenzuela, 2013). Y aunque su uso excesivo puede generar adicción, el uso moderado puede enmarcarse en términos de aprendizaje cooperativo (Muñoz, Fragueiro y Ayuso, 2013). En este sentido, su uso puede incrementarse a través de la creación y consolidación de una política institucional adecuada (De la Hoz, Acevedo y Torres 2015).

Las últimas investigaciones en lengua hispana permiten identificar que, teniendo en cuenta la complejidad de factores en los que se involucran los jóvenes a través de la interacción comunicativa casi siempre bilateral (Barrios, 2014), la construcción de la identidad juvenil en el contexto de la cibercomunicación se ve permeada de oportunidades en términos de aprendizaje, socialización, entretención y mejora de la motivación, así como de riesgos que corresponden al distanciamiento físico, la pérdida de comunicación más asertiva y de la capacidad de escucha (Arab y Díaz, 2015).

Puede considerarse relevante tener en cuenta que un número cada vez mayor de estudiantes utilizan la red y las redes con una finalidad puramente recreativa, aunque piensan que ellas no representan ningún riesgo en su rendimiento académico; de igual manera, la mayoría de ellos rechaza las solicitudes de desconocidos y bloquean sus invitaciones (Toledo y Sánchez, 2012). De esta manera, las alertas sociales, principalmente de la escuela y la familia, actúan de manera persuasiva con los jóvenes.

\section{REFERENCIAS}

Arab, L. E. y Díaz, G. A. (2015). Impacto de las redes sociales e internet en la adolescencia: aspectos positivos y negativos. Revista Médica Clínica Las Condes, 26(1), 7-13. Recuperado de https:// www.sciencedirect.com/science/article/ pii/S0716864015000048

Aparicio, O. Y. (2018). Las TIC como herramientas cognitivas. Revista Interamericana de Investigación, Educación y Pedagogía, RIIEP, 11(1), 67-80. DOI: https://doi. org/10.15332/s1657-107x.2018.0001.07

Aparicio, O. Y. y Ostos, O. L. (2018a). Las TIC como herramientas cognitivas para la investigación. Revista Interamericana de Investigación, Educación y Pedagogía, RIIEP, 11(1), 81-86. DOI: https://doi. org/10.15332/s1657-107x.2018.0001.08

Aparicio Gómez, O. Y. y Ostos Ortiz, O. L. (2018b). El constructivismo y el construccionismo. Revista Interamericana de Investigación, Educación y Pedagogía, RIIEP, 11(2), 115-120. DOI: https://doi. org/10.15332/s1657-107x.2018.0002.05.

Barabási, A. L. (2002) Linked: the new science of networks. Cambridge, MA: Perseus Publishing.

Barrios, G. (2014). La radio escolar como experiencia educativa para construir ciudadanía en las escuelas oficiales del Distrito de Cartagena. Castillo, M. Ponencia llevada a cabo en el $2^{\circ}$ Congreso Internacional de Periodismo Digital, SEAP, Santiago de Chile, Chile.

Bruner, J. S. (1981). Realidad mental y mundos posibles. Madrid, España: Gedisa.

De la Hoz, L. P., Acevedo, D. y Torres, J. (2015). Uso de redes sociales en el proceso de enseñanza y aprendizaje por los estudiantes y profesores de la Universidad Antonio Nariño, Sede Cartagena. Formación universitaria, 8(4), 77-84. DOI: http://dx.doi.org/10.4067/ S0718-50062015000400009. 
Dewey, J. (1938). Experience $\mathcal{E}$ Education. Nueva York: Simon \& Schuster.

Jonassen, D. H. (2000). Computers as mindtools for schools: Engaging critical thinking. New Jersey: Prentice Hall.

Kant, I. (2005). Crítica de la razón pura. México D. F., México: Porrúa.

Kohlberg, L. (1992). Psicología del desarrollo moral. Bilbao, España: Desclée de Bower.

Lalueza, J. L., Crespo, I. y Camps, S. (2008). Las tecnologías de la información y la comunicación y los procesos de desarrollo y socialización. En Coll, C. y Monereo, C. (eds.), Psicología de la educación virtual (pp. 54-73). Madrid, España: Morata. DOI: https://doi.org/10.1080/02147 033.1990.10820936

Muñoz Prieto, M. d. M., Fragueiro Barreiro, M. S. y Ayuso Manso, M. J. (2013). La importancia de las redes sociales en el ámbito educativo. Escuela Abierta, (16), 91-104.

Papert, S. (1982). Desafío a la mente: computadoras y educación. Buenos Aires, Argentina: Galápago.
Pea, R. D. (1985). Beyond amplification: Using the computer to reorganize mental functioning. Educational Psychologist, 20(4), 167-182. DOI: https://doi. org/10.1207/s15326985ep2004_2.

Piaget, J. (1980). Psicología y pedagogía. Barcelona, España: Ariel.

Rheingold, H. (1985). Tools for thought. MIT Press. Recuperado de http://www.rheingold.com/texts/tft/

Siemens, G. (2005). Conectivismo: una teoría de la enseñanza para la era digital. International Journal of Instructional Technology and Distance Learning, 2(10), 3-10.

Toledo, P., y Sánchez, J. (2012). Estudio sobre redes sociales y estudiantes. En Redes educativas: La educación en la sociedad del conocimiento. Sevilla, España: Universidad de Sevilla. DOI: https://doi. org/10.5585/riae.v16i4.2522

Valenzuela, R. (2013). Las redes sociales y su aplicación en la educación. Revista Digital Universitaria, 14(4), 1-14. 